\title{
Distrofia corneana amorfa posterior: relato de caso
}

\author{
Posterior amorphouscornealdystrophy:case report
}

\author{
Lauro Augusto de Oliveira ${ }^{1}$ \\ Luiz Antônio Vieira² \\ Denise de Freitas ${ }^{3}$ \\ Luciene Barbosa de Sousa ${ }^{4}$
}

\begin{tabular}{l} 
RESUMO \\
\hline O objetivo deste trabalho é alertar o oftalmologista da possibilidade de se \\
deparar com casos raros de distrofias corneanas. Neste caso correlacio- \\
namos os achados clínicos da distrofia amorfa posterior com refração, \\
topografia e biomicroscopia ultra-sônica.
\end{tabular}

Descritores: Distrofias hereditárias da córnea; Opacidade da córnea/ultra-sonografia; Estroma corneal; Acuidade visual; Córnea/patologia; Linhagem; Relatos de casos [tipo de publicação]

\section{INTRODUĈ̃̃ O}

Distrofias corneanas são um grupo de desordens não inflamatórias, de herança genética determinada, caracterizadas por opacidades corneanas de aparência clínica diversa e também pela sua evolução e tratamento ${ }^{(1)}$. A distrofia corneana amorfa posterior foi descrita por Carpel et al., em 1977(2). É uma condição de herança autossômica dominante determinada por opacidades corneanas localizadas no estroma posterior.

Dentre os raros casos descritos foram identificados opacificações branco-acinzentadas no estroma posterior e afinamento corneano ${ }^{(3)}$.

Córneas aplanadas, hipermetropia, alterações na superfície e no estroma irianos, processos irianos se estendendo até a linha de Schwalbe e extensão da opacidade até o limbo também já foram descritos ${ }^{(4)}$.

Neste estudo descrevemos um caso de distrofia corneana amorfa posterior e fazemos uma revisão da literatura nacional e internacional.

Trabalho realizado no Setor de Doenças Externas e Córnea do Departamento de Oftalmologia da Universidade Federal de São Paulo - UNIFESP.

Pós-graduando (nível Doutorado) do Setor de Doenças Externas e Córnea do Departamento de Oftalmologia da Universidade Federal de São Paulo - UNIFESP São Paulo (SP) - Brasil.

${ }^{2}$ Doutor em Oftalmologia da UNIFESP - São Paulo (SP) - Brasil.

${ }^{3}$ Livre Docente em Oftalmologia da UNIFESP - São Paulo (SP) - Brasil.

${ }^{4}$ Doutora em Oftalmologia da UNIFESP - São Paulo (SP) - Brasil.

Endereço para correspondência: Lauro Augusto de Oliveira. Av. Onze de Junho, 685 - São Paulo (SP) CEP 04041-052

E-mail: laopadilha@terra.com.br.

Recebido para publicação em 04.04.2005

Última versão recebida em 30.09.2005

Aprovação em 08.06.2006

Nota Editorial: Depois de concluída a análise do artigo sob sigilo editorial e com a anuência do Dr. Ricardo Lewinsky sobre a divulgação de seu nome como revisor, agradecemos sua participação neste processo.

\section{RELATO DE CASO}

Paciente de 6 anos, feminino, com acompanhante referindo mancha em forma de meia lua, de progressão lenta, em ambos os olhos há 3 anos. Acuidade visual sem correção era de 20/100 em ambos os olhos (paciente mal informante). Acuidade visual com a melhor correção era de 20/70 no OD e 20/100 no OE (plano -2,00 DC X $10^{\circ}$ e plano -3,00 DC x $160^{\circ}$ ).

$\mathrm{Na}$ biomicroscopia apresentava aumento da espessura corneana que preservava apenas região paracentral inferior, localização coincidente com área suspeita de ectasia. Apresentava uma linha de opacidade branco-acinzentada no estroma posterior que também preservava a região paracentral inferior em ambos os olhos (Figura 1). Sensibilidade corneana era normal.

A topografia corneana (23/08/00, EyeSys System 2000S, Houston, TX, U.S.A.) demonstrou um astigmatismo irregular e assimétrico em ambos os olhos, porém mais evidente no olho esquerdo. Percebe-se um aumento da curvatura corneana na região paracentral nasal inferior bilateral (OD: 42,86; OE: 43,73) que coincide com a área mais fina detectada pela biomicroscopia 


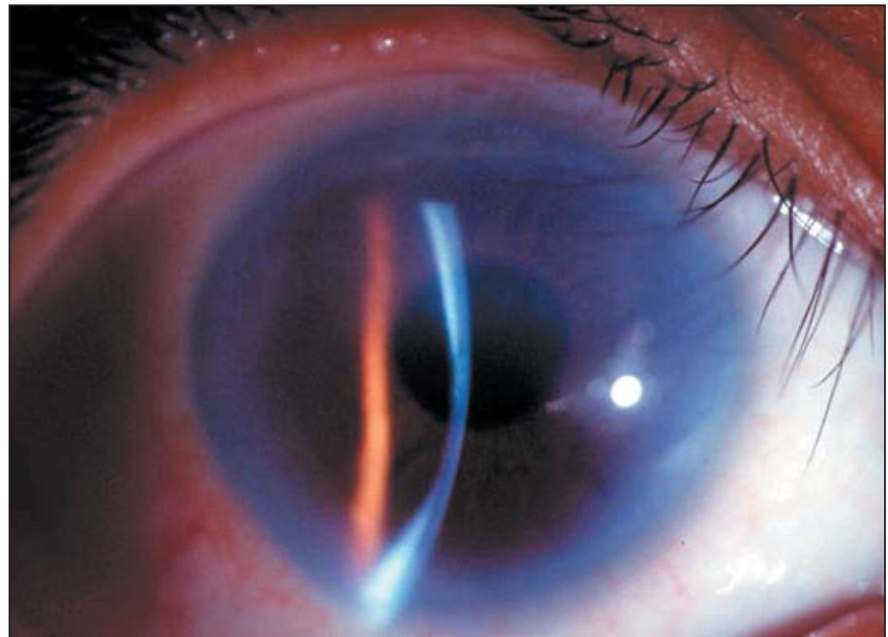

Figura 1 - Biomicroscopia OD mostrando opacidade estromal posterior e área de afinamento

ultra-sônica. Demonstrou também valores ceratométricos próximos de 40,00 dioptrias (córneas aplanadas) (Figura 2). A biomicroscopia ultra-sônica (Ultrasound Biomicroscope model 840, Humphrey Instruments Inc., Dublin, CA, U.S.A.) revelou aumento da espessura corneana nas regiões mais periféricas (1088 $\mu \mathrm{m}$ em OD e $1100 \mu \mathrm{m}$ em OE), com discreto afinamento $(470 \mu \mathrm{m})$ na região paracentral nasal inferior de ambos os olhos. Evidenciou também uma opacidade linear em estroma profundo (Figura 3).

A investigação familiar (heredograma) só foi realizada na mãe da paciente, a qual não apresentou nenhuma alteração corneana. Os demais membros (irmão e pai) não foram submetidos ao exame oftalmológico por motivos familiares e socioeconômicos.

A investigação para tuberculose (PPD) e sorológica para sífilis foram negativas.

Como tratamento, prescrevemos a correção óptica e optamos por uma conduta expectante. Tentamos adaptação de lentes de contato e obtivemos melhora da acuidade visual, porém a paciente e sua mãe se opuseram ao uso das lentes prescritas. Desde então foi orientado retorno semestral para reavaliação oftalmológica geral, exame refracional e fotodocumentação.

\section{DISCUSSÃO}

A distrofia amorfa posterior é uma condição bastante rara mesmo nos grandes centros de referência oftalmológica(3). Existem poucas publicações na literatura sobre esta patologia, dentre as quais, todas são descrições de achados em relatos de casos. Dentre os casos relatados pelo Brasil, talvez este seja o primeiro descrito na literatura nacional. O diagnóstico de patologias de baixa incidência e prevalência como raras distrofias corneanas é muitas vezes lembrado por exclusão e mesmo assim podem ser questionados. Algumas distrofias e disgenesias corneanas congênitas compartilham vários acha-

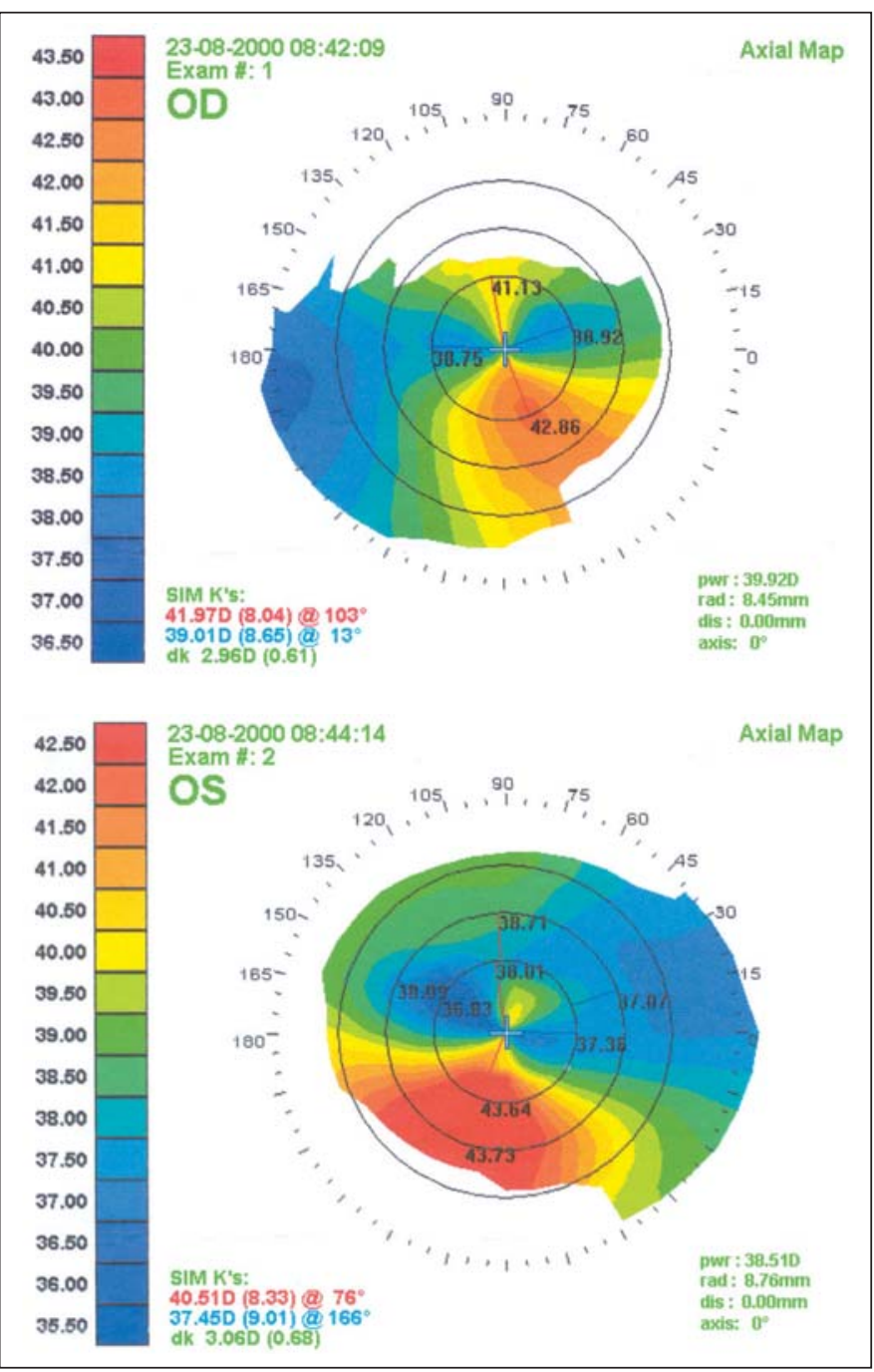

Figura 2 - Topografias mostrando aumento da curvatura em região paracentral nasal inferior em ambos os olhos

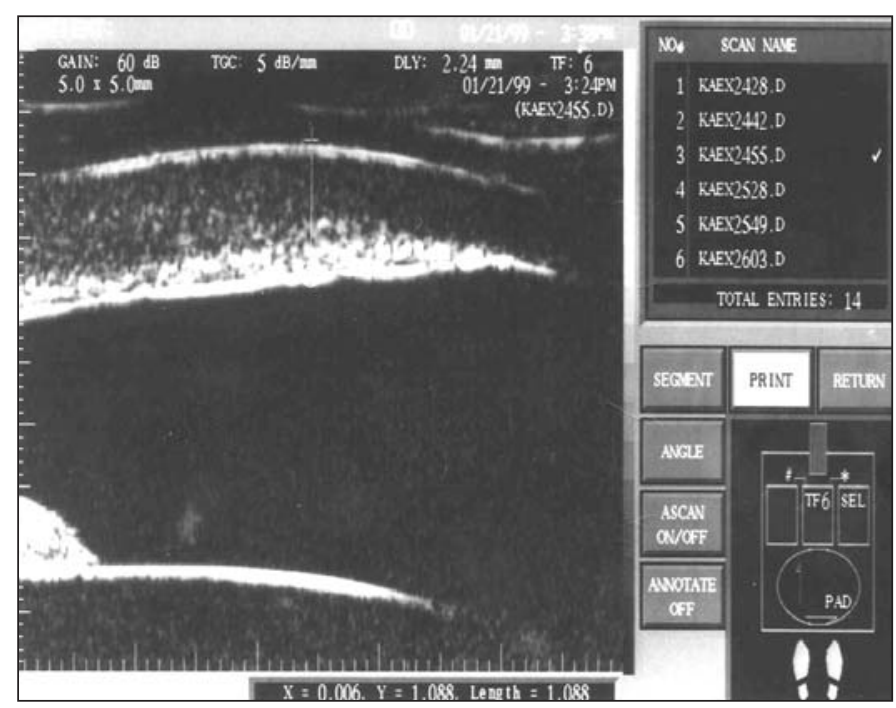

Figura 3 - UBM mostrando aumento da espessura corneana e opacidade em estroma profundo 
dos: podem estar presentes ao nascimento; podem ser herdadas, normalmente em um padrão dominante; anormalidades corneanas estão presentes e também podemos encontrar alterações em outros tecidos de origem mesenquimal como íris e ângulo da câmara anterior como na distrofia polimorfa posterior e anomalia de Peters; normalmente são bilaterais. Ainda como diagnóstico diferencial devemos incluir a distrofia endotelial hereditária congênita (CHED).

Moshegov et al., estudaram nove pacientes de três gerações de uma mesma família e tentaram classificar esta distrofia em duas formas distintas de expressão: centroperiférica e periférica. Todos pacientes com a forma centroperiférica apresentavam hipermetropia, valores ceratométricos abaixo de 41,00 dioptrias e com espessura central menor que $0,500 \mathrm{~mm}^{(5)}$. Como a paciente estudada apresentou apenas uma área corneana livre (paracentral nasal inferior), leitura ceratométrica média abaixo de 40,00 dioptrias e um ligeiro afinamento inferior, acreditamos que a mesma se enquadraria na forma centroperiférica proposta acima. Da mesma forma, Dunn et al., ao descreverem oito pacientes de cinco gerações de uma mesma família notaram as seguintes características: hipermetropia, córneas planas e anormalidades irianas ${ }^{(4)}$. No caso em questão não encontramos nenhuma anormalidade iriana óbvia. Em outro estudo que abrangeu sete casos os autores incluíram os seguintes achados clínicos neste tipo de distrofia: variações na espessura corneana observadas na biomicroscopia e também na paquimetria ultra-sônica, astigmatismo corneano irregular e ectasia progressiva ${ }^{(6)}$.

Assim como descreveram alguns autores, na biomicroscopia ultra-sônica corneana desta paciente encontramos opacidades estromais profundas e uma área de afinamento paracentral $^{(1)}$.

Devido à raridade desta patologia é interessante que os oftalmologistas se atentem para esta hipótese quando estiverem diante de um quadro de opacidade estromal posterior bilateral. É relevante também examinar os membros da família destes pacientes o quanto antes, com a preocupação em detectar opacidades e/ou ametropias que possam levar a ambliopia.

\section{ABSTRACT}

The purpose of this paper is to warn the ophthalmologist about the possibility of facing rare cases of corneal dystrophies. Clinical findings of a case of posterior amorphous dystrophy were correlated with refraction, topography, and ultrasound biomicroscopy.

Keywords: Corneal dystrophies, hereditary; Corneal opacity/ ultrasonography; Corneal stroma; Visual acuity; Cornea/pathology; Pedigree; Case reports [publication type]

\section{REFERÊNCIAS}

1. Castelo Branco B, Chalita MR, Casanova FH, Branco AB, Allemann N, Freitas D. Posterior amorphous corneal dystrophy: ultrasound biomicroscopy findings in two cases. Cornea. 2002;21(2):220-2.

2. Carpel EF, Sigelman RJ, Doughman DJ. Posterior amorphous dystrophy. Am J Ophthalmol. 1977;83(5):629-32.

3. Roth SI, Mittelman D, Stock EL. Posterior amorphous corneal dystrophy: An ultrastructural study of a variant with histopatological features of an endothelial dystrophy. Cornea. 1992;11(2):165-72.

4. Dunn SP, Krachmer JH, Ching SS. New findings in posterior amorphous corneal dystrophy. Arch Ophthalmol. 1984;102(2):236-9.

5. Moshegov CN, Hoe WK, Wiffen SJ, Daya SM. Posterior amorphous corneal dystrophy. A new pedigree with phenotypic variation. Ophthalmology. 1996; 103(3):474-8.

6. Grimm BB, Waring GO 3rd, Grimm SB. Posterior amorphous corneal dysgenesis. Am J Ophthalmol. 1995;120(4):448-55. 